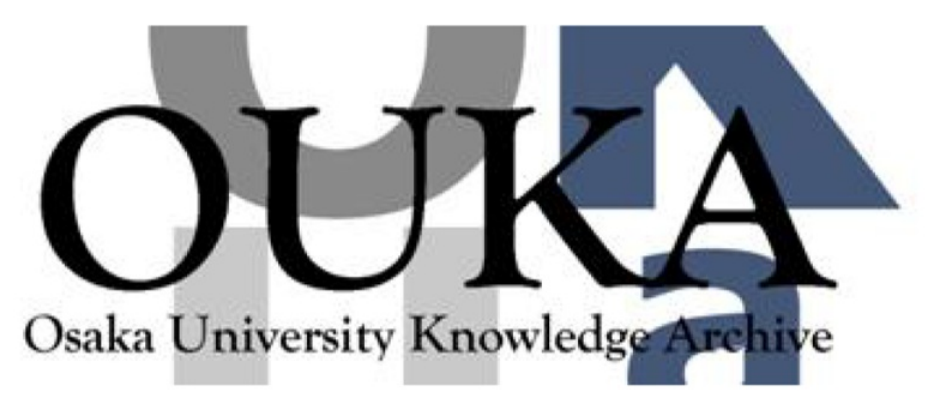

\begin{tabular}{|c|l|}
\hline Title & $\begin{array}{l}\text { Analysis of flexural mode focusing by a } \\
\text { semianalytical finite element method }\end{array}$ \\
\hline Author(s) & $\begin{array}{l}\text { Hayashi, Takahiro; Kawashima, Koichiro; Sun, } \\
\text { Zongqi et al. }\end{array}$ \\
\hline Citation & $\begin{array}{l}\text { The Journal of the Acoustical Society of } \\
\text { America. 113(3) p. 1241-p.1248 }\end{array}$ \\
\hline Issue Date & $2003-03$ \\
\hline oaire:version & VoR \\
\hline URL & $\begin{array}{l}\text { https://hdl. handle. net/11094/84502 } \\
\text { Copyright 2003 Acoustical Society of America. } \\
\text { This article may be downloaded for personal use } \\
\text { only. Any other use requires prior permission } \\
\text { of the author and the Acoustical Society of } \\
\text { America. }\end{array}$ \\
\hline rights \\
\hline Note & \begin{tabular}{l} 
\\
\hline
\end{tabular} \\
\hline
\end{tabular}

Osaka University Knowledge Archive : OUKA

https://ir. Library. osaka-u. ac. jp/

0saka University 


\title{
Analysis of flexural mode focusing by a semianalytical finite element method
}

\author{
Takahiro Hayashi ${ }^{\mathrm{a})}$ and Koichiro Kawashima \\ Department of Mechanical Engineering, Nagoya Institute of Technology, Gokiso Showa Nagoya 466-8555, \\ Japan
}

Zongqi Sun and Joseph L. Rose ${ }^{\text {b) }}$

Department of Engineering Science and Mechanics, The Pennsylvania State University, 411 E Earth \& Engineering Science Building, University Park, Pennsylvania 16802

(Received 24 May 2002; revised 13 December 2002; accepted 16 December 2002)

\begin{abstract}
Focusing is one of the most promising techniques for the detection of small defects in pipe works, in which guided waves including longitudinal and flexural modes are tuned so that ultrasonic energy can be focused at a target point in a pipe, and analysis of reflected waves gives information on defects such as location and size. In this paper, the focusing technique is discussed by way of a simulation of guided wave propagation in pipe by a semianalytical finite element method (SAFE). Experiments and SAFE calculations were compared for waveforms transmitted by a single transducer and received at different circumferential positions initially, and then the focusing phenomena were experimentally observed using focusing parameters obtained by calculations. Calculation and visualization were conducted to clarify focusing phenomena in pipe in investigating the potential of the focusing technique. These results show that the time-reversal idea helps in understanding focusing and that resolution of focusing is strongly affected by incident waveforms as well as the number of channels available in an experiment. (C) 2003 Acoustical Society of America. [DOI: 10.1121/1.1543931]
\end{abstract}

PACS numbers: 43.20.Gp, 43.20.Px [LLT]

\section{INTRODUCTION}

Since thousands of miles of tubing and piping are used in all sorts of power generation, petrochemical, and manufacturing plants, nondestructive evaluation has become an important subject for reduced inspection time and costs. Ultrasonic nondestructive evaluation by guided waves propagating over long distances in a pipe has recently attracted considerable attention as a useful means for solving these issues; many theoretical and experimental studies have been published. $^{1-11}$

All of these works on guided waves in a pipe are based on a theoretical study of the normal mode expansion presented by Gazis in $1959,{ }^{12}$ in which dispersion curves for a hollow cylinder with outer- and inner traction-free surfaces were derived and fundamental useful knowledge was presented on guided waves in a pipe. In order to achieve our aim of practical applications to quantitative evaluation for cracks in a pipe, however, more detailed theoretical or numerical studies have been required. The focusing technique, especially, proposed by $\mathrm{Li}$ and $\mathrm{Rose}^{3,4}$ is expected to represent a key technique for defect detection in a pipe. The approach, however, is very hard to understand intuitively from the initial fundamental theoretical studies.

The basic idea of the focusing technique is as follows. First, a displacement distribution in the circumferential direction (circumferential profile) is predicted theoretically for partial loading by a single transducer. Circumferential pro-

\footnotetext{
${ }^{a)}$ Electronic mail: hayashi@megw.mech.nitech.ac.jp

b)Electronic mail: jlresm@engr.psu.edu
}

files for multiple transducers can then be expressed by a superposition of this single transducer profile. Then, by tuning amplitudes and time delays of each channel independently, waveforms are enhanced at some predetermined target in a pipe. Since ultrasonic energy is converged on the target, a large reflected echo can be detected only when a defect is located at the target. Scanning various targets can take place by changing amplitudes and time delays. Observation of reflected waves then provides useful information on defects in a pipe, such as location and size.

The purpose of this study is to analyze this focusing technique by way of simulation and visualization of guided wave propagation.

Numerical calculation modeling such as finite element method (FEM) and boundary element method (BEM) is suitable for simulating ultrasonic wave propagation and to investigate it in detail. Moreover, visualizing these calculation results helps our understandings in such complicated wave propagation as guided waves. The semianalytical finite element method (SAFE), in which wave propagation in the longitudinal and circumferential direction is expressed by a superposition of orthogonal harmonics, is one of the most effective modeling techniques for carrying out large computations for guided wave propagation in a pipe. This special modeling technique designed for bar- and plate-like objects was developed by Cheng ${ }^{13}$ and Zienkiewicz. ${ }^{14}$ Recently, Datta $^{15-18}$ and Dong ${ }^{19,20}$ have made significant progress on guided waves using SAFE. The author ${ }^{21}$ have studied complicated Lamb waves propagating in laminated plates using calculation and visualization techniques.

In this paper, the SAFE is used for simulation of cylin- 
drical guided wave propagation. First, calculated waveforms are compared with experiments to confirm the accuracy of the calculations and SAFE calculations are applied to focusing experiments. Next, we investigate the focusing status and its application to defect detection from visualization results. Moreover, the potential values of the focusing technique are discussed.

\section{PRELIMINARY}

\section{A. Semianalytical FEM}

In the SAFE used here, waveforms in circumferential $(\theta)$ and longitudinal $(z)$ directions represent orthogonal harmonic functions, and the analytical dimensions are then reduced into one dimension in the thickness direction $(r)$. Therefore, even guided wave propagation over long distance can be calculated with short calculation times and a small computational memory. Since the basic formulations are written in the references ${ }^{14-18}$ in detail, only some fundamental and additive equations will be represented in this section. Displacement vector $\mathbf{U}$, containing displacements at nodes divided in the $r$ direction, is represented using orthogonal harmonic functions $\exp (\operatorname{in} \theta), \exp (i \xi z)$, and $\exp (-i \omega t)$ in the $r, \theta$, and $t$ (time) direction, respectively

$$
\begin{aligned}
& \mathbf{U}(\theta, z, t)=\int_{-\infty}^{+\infty} \overline{\mathbf{U}}(\theta, z) \exp (-i \omega t) d \omega, \\
& \overline{\mathbf{U}}(\theta, z)=\sum_{n=-\infty}^{+\infty} \overline{\mathbf{U}}_{n}(z) \exp (i n \theta), \\
& \overline{\mathbf{U}}_{n}(z)=\int_{-\infty}^{+\infty} \tilde{\mathbf{U}}_{n} \exp \left(i \xi_{n} z\right) d \xi,
\end{aligned}
$$

where $\overline{\mathbf{U}}$ denotes the Fourier transform of $\mathbf{U}$ in terms of time $t$, and means the complex amplitude for harmonic waves at the angular frequency $\omega . \overline{\mathbf{U}}_{n}$ is a complex Fourier series of $\overline{\mathbf{U}}$ in terms of circumferential direction $\theta, \tilde{\mathbf{U}}_{n}$ is the Fourier transform of $\overline{\mathbf{U}}_{n}$ in terms of longitudinal direction $z$, and $\xi_{n}$ is the wave number propagating into the $\pm z$ directions for the $n$th circumferential function.

Similar to ordinary FEM, the virtual work principle or Hamilton's principle leads to the governing equations. The insertion of Eq. (1) in the governing equations gives eigensystems for the $n$th circumferential harmonic. Solving this eigensystem yields $2 M$ eigenvalues $\xi_{n m}$ and $2 M$ right eigenvectors $\Phi_{n m}^{R}$ (2M vector), where $M$ is the dimension of the nodal displacement vector $U$ and three times the number of nodes, and $m=1,2, \ldots, 2 M$. The eigenvalue $\xi_{n m}$ corresponds to the wave number of the $m$ th mode and $n$th family. When it is real, it means a propagating mode, and an evanescence mode is represented for complex $\xi_{n m}$. Calculating the eigenvalues $\xi_{n m}$ at many frequency steps yields dispersion curves for the $n$th family.

Here, following Ref. 19, the displacement vector $\overline{\mathbf{U}}_{n}$ for the $n$th circumferential harmonic ( $n$th family) is obtained as

$$
\overline{\mathbf{U}}_{n}=\sum_{m=1}^{M} \alpha_{n m} \Phi_{n m}^{R \text { upper }} \exp \left(i \xi_{n m} z\right),
$$

where $\alpha_{n m}$ is an amplitude of the $(n, m)$ mode to be obtained from boundary conditions, and $\Phi_{n m}^{R \text { upper }}$ is the vector consisting of upper $M$ elements of $\Phi_{n m}^{R}$, representing displacement distribution in the $r$ direction. Equation (4) exhibits that $\overline{\mathbf{U}}_{n}$ is described as a superposition of guided wave modes for the $n$th circumferential harmonic.

Now suppose that a line source is located at $z=z_{s},|\theta|$ $<\theta_{0}$ [Fig. 1(a)] and that the displacement vector is described as

$$
\overline{\mathbf{U}}_{n}^{\text {lineS }}=\sum_{m=1}^{M} \alpha_{n m}^{\text {lineS }} \Phi_{n m}^{R \text { upper }} \exp \left\{i \xi_{n m}\left(z-z_{s}\right)\right\} .
$$

This solution has been obtained in Ref. 19. Then, the solution for the angle wedge transducer of incident angle $\theta_{i}$ and located at $|\theta|<\theta_{0},\left|z-z_{s}\right|<z_{0}$ can be obtained as an integration of Eq. (5). See Fig. 1(b). By considering the phase shift due to incident angle, only amplitude $\alpha_{n m}$ should be replaced as

$$
\alpha_{n m} \rightarrow \alpha_{n m} \times \frac{2 \sin \left\{\left(\xi_{0}-\xi_{n m}\right) z_{0}\right\}}{\xi_{0}-\xi_{n m}},
$$

where $\xi_{0}$ is represented as $\xi_{0}=\xi_{a} \sin \theta_{i}$ when assuming the wave number in the wedge is $\xi_{a}$.

When the angle wedge receiver is the same as the transmitter with the width $2 \theta_{0}$ and $2 z_{0}$ in the $\theta$ and $z$ directions, respectively, the amplitude of the received signal is obtained as

$$
\alpha_{n m} \rightarrow \alpha_{n m} \times \frac{2 \sin \left\{\left(\xi_{n m}-\xi_{0}\right) z_{0}\right\}}{\xi_{n m}-\xi_{0}} \times \frac{2 \sin n \theta_{0}}{n} .
$$

When the excitation point is $\theta=\theta_{s}$ instead of $\theta=0$

$$
\alpha_{n m} \rightarrow \alpha_{n m} \times \exp \left(-i n \theta_{s}\right)
$$

gives the solutions.

To summarize, when the angle wedge transmitter with incident angle $\theta_{i}$ and width of $2 z_{0}$ and $2 \theta_{0}$ are installed at $z=z_{s}, \theta=\theta_{s}$, displacements at the angle wedge receiver with receiving angle $\theta_{i}$ and width of $2 z_{0}$ and $2 \theta_{0}$ [Fig. 1(c)] are given as

$$
\begin{aligned}
\overline{\mathbf{U}}_{n}^{\text {angle } S+\text { angle } R}= & \sum_{m=1}^{M} \alpha_{n m}^{\text {angle } S+\text { angle } R} \Phi_{n m}^{R} \text { upper } \\
& \times \exp \left\{i \xi_{n m}\left(z-z_{s}\right)\right\}, \\
\alpha^{\text {angle } S+\text { angle } R}= & \alpha^{\operatorname{line} S} \times \frac{2 \sin \left\{\left(\xi_{0}-\xi_{n m}\right) z_{0}\right\}}{\xi_{0}-\xi_{n m}} \\
& \times \frac{2 \sin \left\{\left(\xi_{n m}-\xi_{0}\right) z_{0}\right\}}{\xi_{n m}-\xi_{0}} \times \frac{2 \sin n \theta_{0}}{n} \\
& \times \exp \left(-i n \theta_{s}\right) .
\end{aligned}
$$

\section{B. Focusing algorithm}

$N$ transmitters and $N$ receivers are located in the circumferential direction and numbered $1,2, \ldots, N$ from the top of the pipe as shown in Fig. 2. Then, the complex amplitude of a harmonic wave at the frequency $\omega$ traveling from the $i$ th transmitter to the $j$ th receiver is defined as $H_{i j} . H_{i j}$ is pre- 


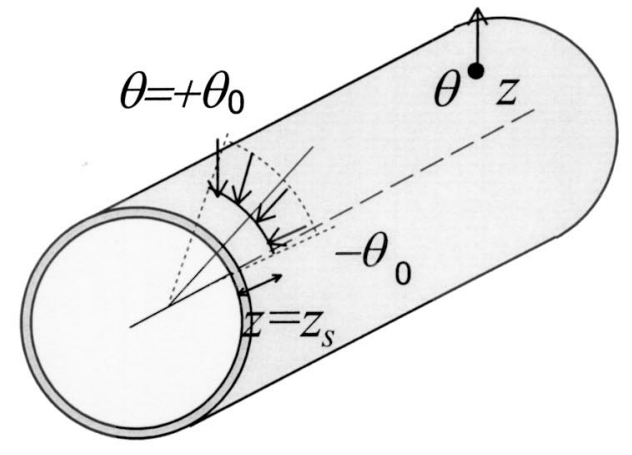

(a) Line source and point receiver.

Displacement field is described by Eq.(5).

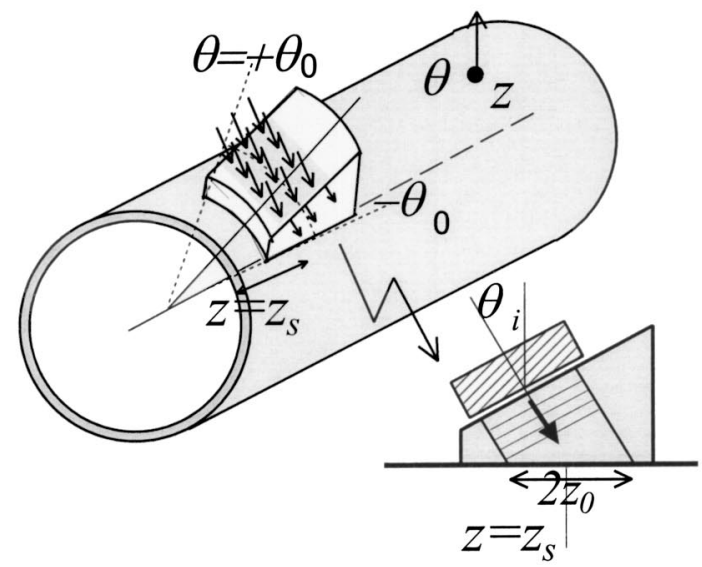

(b) Angle wedge transmitter and point receiver. Displacement field is described in Eq.(6).

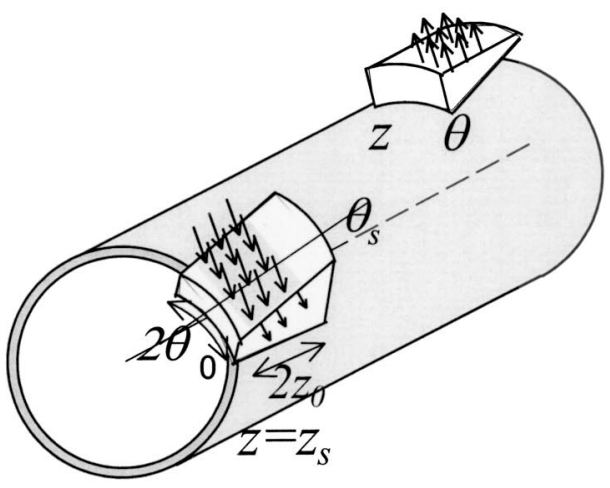

(c) Angle wedge transmitter and angle wedge receiver Displacement field is described by Eq. (9)

FIG. 1. Boundary conditions.

determined by experiments or calculations, containing the information of amplitude and phase. Controlling the output level (amplitude) $A_{i}$ and time delay $t_{i}$ of the $i$ th transmitter, the received signal is described as $a_{i} H_{i j}$, where

$$
a_{i}=A_{i} \exp \left(-i \omega t_{i}\right) .
$$

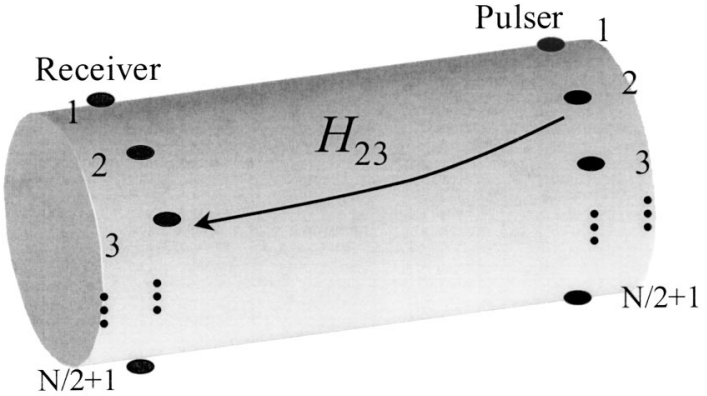

FIG. 2. Focusing algorithm.

Tuning all amplitudes and time delays from 1 to $N$, signals at the $j$ th receiver $q_{j}$ can be described as a superposition of $a_{i} H_{i j}$ as

$$
q_{j}=\sum_{i=1}^{N} a_{i} H_{i j} .
$$

This is represented in the matrix form as

$$
\begin{aligned}
& \mathbf{q}=\mathbf{H a}, \\
& \mathbf{q}=\left[\begin{array}{lllll}
q_{1} & q_{2} & q_{3} & \cdots & q_{N}
\end{array}\right]^{T}, \quad \mathbf{a}=\left[\begin{array}{lllll}
a_{1} & a_{2} & a_{3} & \cdots & a_{N}
\end{array}\right]^{T} .
\end{aligned}
$$

Here, letting the received signals be

$$
\mathbf{q}=\left[\begin{array}{lllll}
1 & 0 & 0 & \cdots & 0
\end{array}\right]^{T},
$$

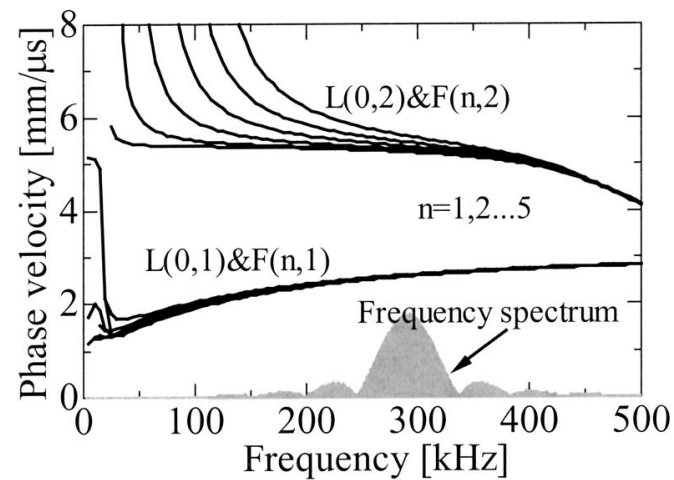

(a) Phase velocity

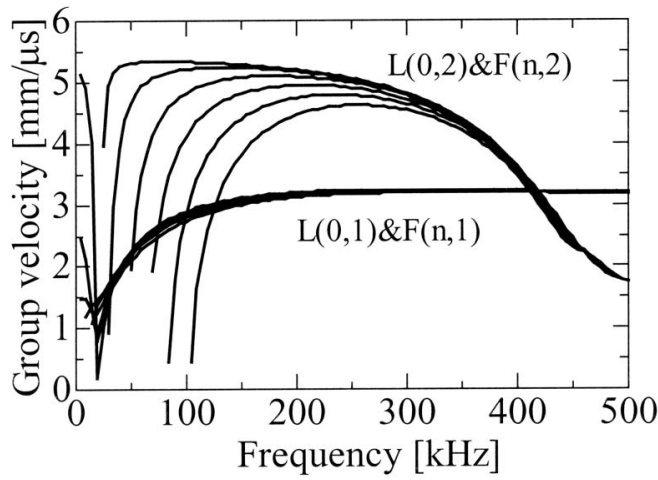

(b) Group velocity

FIG. 3. Dispersion curves for a steel pipe used in this paper; $88-\mathrm{mm}$ outer diameter and 5-mm thickness. (Gray region denotes the frequency spectrum of incident wave, $290 \mathrm{kHz}, 7.5$-cycle tone-burst waves.) 


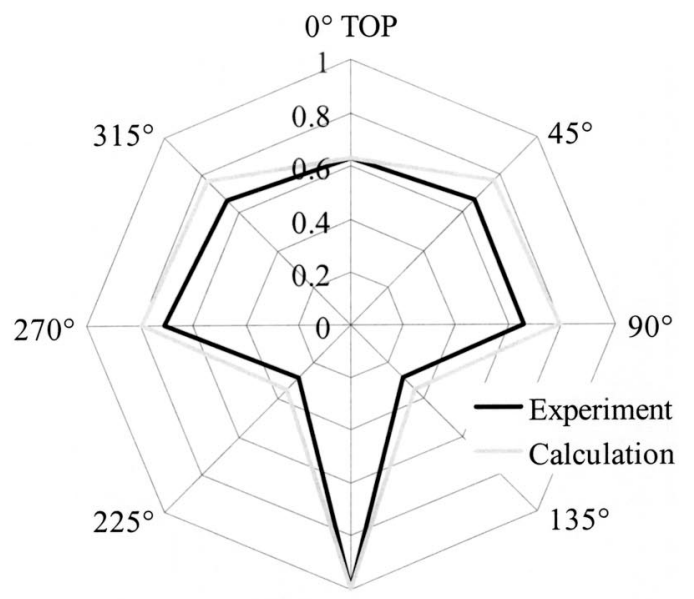

$180^{\circ} \mathrm{BOTTOM}$

(a) Circumferential profile

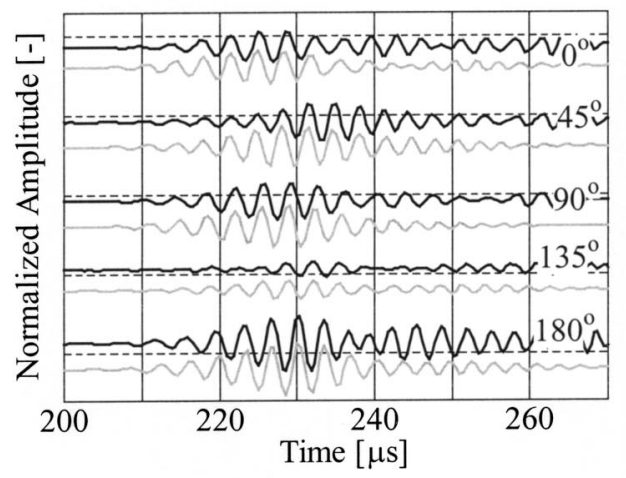

(b) Time domain profiles

FIG. 4. Experimental (black line) and calculated (gray line) waveforms at different circumferential positions at $\mathrm{L}=800 \mathrm{~mm}$.

then we have a complex amplitude

$$
\mathbf{a}=\mathbf{H}^{-1} \mathbf{q} \text {. }
$$

Complex amplitude components $a_{i}$ are represented by amplitudes and time delays as shown in Eq. (10).

From the predefined target amplitude $\mathbf{q}$ and the matrix $\mathbf{H}$ determined by numerical calculations, necessary amplitudes and time delays for each element are calculated. When a pipe in question is geometrically symmetric like a straight homogeneous pipe, matrix $\mathbf{H}$ becomes a cyclic matrix as

$$
\begin{aligned}
& H_{11}=H_{22}=H_{33}=\cdots=H_{N N}, \\
& H_{12}=H_{23}=H_{34}=\cdots=H_{N 1}, \\
& \cdots,
\end{aligned}
$$

and then the deriving Eq. (14) is equivalent to the convolution algorithm developed by $\mathrm{Li}^{4}$

\section{COMPARISON OF SAFE CALCULATION AND EXPERIMENT}

The following two experiments were carried out in this section in order to verify the SAFE calculations. The first one was a comparison between experimental and calculated

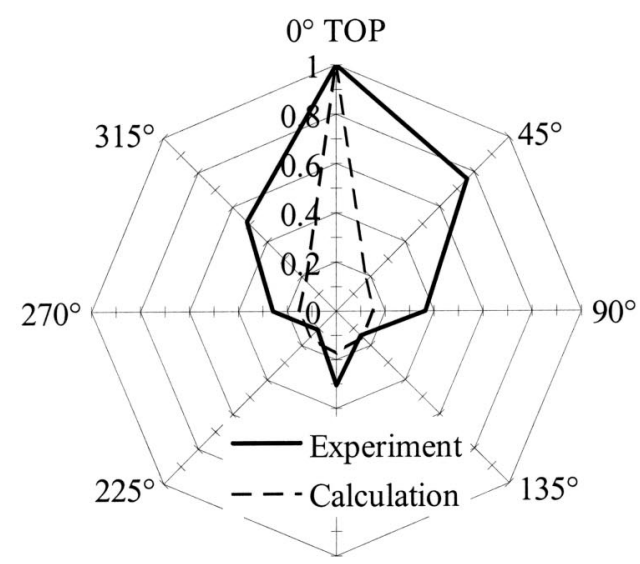

$180^{\circ}$ BOTTOM

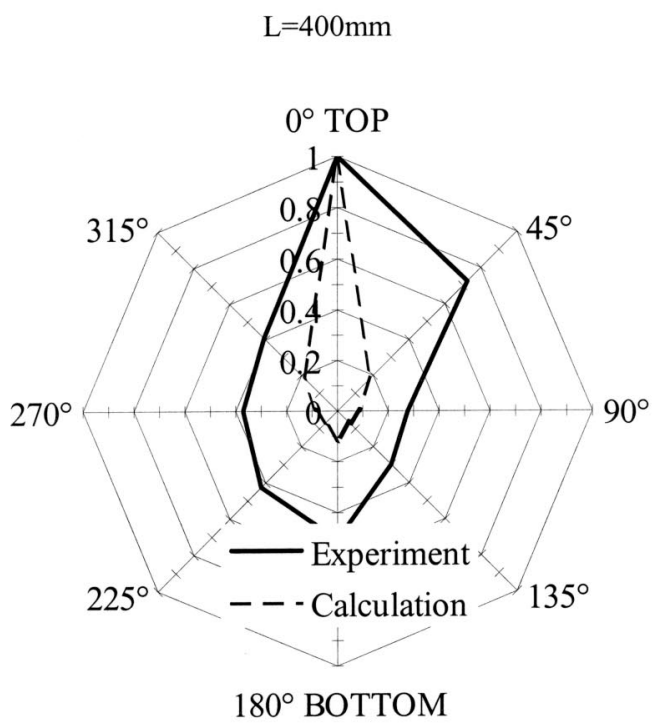

$\mathrm{L}=800 \mathrm{~mm}$

FIG. 5. Circumferential profile at focal points of $\mathrm{L}=400 \mathrm{~mm}$ and $\mathrm{L}=800$ $\mathrm{mm}$.

flexural modes that were exited by a single transducer and received at various circumferential positions. The second one was a confirmation of the focusing phenomena by using amplitudes and time delays obtained from the SAFE calculations of circumferential profile $H_{i j}$ and the focusing algorithm.

In these experiments, a steel pipe of $88-\mathrm{mm}$ diameter and 5-mm thickness is used. Eight angle wedge transmitters with an incident angle of $30^{\circ}$ are fixed at the end of the pipe and an angle wedge receiver with $30^{\circ}$ receiving angle can be moved to receive waveforms at different circumferential and longitudinal positions.

Figure 3 shows dispersion curves of the steel pipe used in these experiments as well as a frequency spectrum of incident signals of about $290 \mathrm{kHz}$ center frequency. L and $\mathrm{F}$ in Fig. 3 denote Longitudinal and Flexural modes, respectively. The numbers in parentheses correspond to $(n, m)$ in Eq. (4), representing the $m$ th mode of the $n$th family. The incident and receiving angles of $30^{\circ}$ are suitable for exciting and 
TABLE I. Amplitude and time-delay computation for focusing at the top for $\mathrm{L}=400 \mathrm{~mm}$ and $800 \mathrm{~mm}$.

\begin{tabular}{|c|c|c|c|c|c|c|c|c|}
\hline $\begin{array}{c}\mathrm{L}=400 \mathrm{~mm} \\
\text { Top }\end{array}$ & $\begin{array}{c}\text { Top, } \\
0^{\circ}\end{array}$ & $45^{\circ}$ & $90^{\circ}$ & $135^{\circ}$ & $\begin{array}{c}\text { Bottom, } \\
180^{\circ}\end{array}$ & $225^{\circ}$ & $270^{\circ}$ & $315^{\circ}$ \\
\hline Amplitude [-] & 1.00 & 0.98 & 0.92 & 0.87 & 0.83 & 0.87 & 0.92 & 0.98 \\
\hline $\begin{array}{l}\text { Time delay } \\
{[\mu \mathrm{s}]}\end{array}$ & 2.38 & 2.04 & 1.22 & 0.00 & 1.88 & 0.00 & 1.22 & 2.04 \\
\hline $\begin{array}{c}\mathrm{L}=800 \mathrm{~mm} \\
\text { Top }\end{array}$ & $\begin{array}{c}\text { Top, } \\
0^{\circ}\end{array}$ & $45^{\circ}$ & $90^{\circ}$ & $135^{\circ}$ & $\begin{array}{c}\text { Bottom, } \\
180^{\circ}\end{array}$ & $225^{\circ}$ & $270^{\circ}$ & $315^{\circ}$ \\
\hline Amplitude [-] & 0.48 & 0.60 & 0.72 & 0.35 & 1.00 & 0.35 & 0.72 & 0.60 \\
\hline $\begin{array}{l}\text { Time delay } \\
{[\mu \mathrm{s}]}\end{array}$ & 1.78 & 2.52 & 1.39 & 1.50 & 0.00 & 1.50 & 1.39 & 2.52 \\
\hline
\end{tabular}

detecting $\mathrm{L}(0,2)$ and $\mathrm{F}(n, 2)$ modes. In the first single transmitter tests, waveforms at eight circumferential positions $\left(0^{\circ}, 45^{\circ}, 90^{\circ}, 135^{\circ}, 180^{\circ}, 225^{\circ}, 270^{\circ}, 315^{\circ}\right)$ were detected in through-transmission configurations with a fixed angled transmitter on the top $\left(0^{\circ}\right)$ of the pipe and a receiving transducer at different positions. In the second focusing tests, a Matec multichannel system independently tuned amplitudes and time delays for eight angle wedge transmitters located at regular intervals in the circumferential direction. Circumferential profiles were measured by scanning the receiving transducer in the circumferential direction.

In the SAFE calculations, solving the eigensystems determined by given material properties and geometries yields wave numbers $\xi_{n m}$ and wave structure vectors $\Phi_{n m}^{R}$ upper in Eq. (4), and the boundary conditions give amplitudes $\alpha_{n m}$. Then, displacements in Eq. (4) were given for each circumferential harmonic $n$ and frequency step $\omega$. Waveforms at any points $\mathbf{U}(\theta, z, t)$ are obtained by substituting displacements Eq. (4) into Eq. (2) and then into Eq. (1). Tone-burst signals of $290 \mathrm{kHz}$ and 7.5 cycles were adopted as an excitation stress on the surface of the pipe so as to meet experimental conditions. So, boundary conditions in these calculations are traction-free on both inner and outer surface of the pipe and tone-burst normal stress applied on the loading region. The other geometric parameters such as transducer width $\left(z_{0}\right.$ $\left.=7.5 \mathrm{~mm}, \theta_{0}=22.5^{\circ}\right)$ and transducer angle $\left(\theta_{i}=30^{\circ}\right)$ were adjusted to the experiments. The pipe was divided by eight quadratic cylindrical elements in the $r$ direction as in Ref. 19. Calculations were done for 11 circumferential harmonics from $n=-5$ to 5 and for 1000 frequency steps from 0 to 2.0 $\mathrm{MHz}$.

Figure 4 shows the comparisons between experimental and computated waveforms (displacements) when the intertransducer distance $L$ was $800 \mathrm{~mm}$. Figure 4(a) presents the distribution of maximum amplitude of the waveforms detected at various circumferential positions (circumferential profile). Figure 4(b) shows waveforms obtained both experimentally and calculated at various circumferential positions.
Since the waveforms at the circumferential position of $225^{\circ}$, $270^{\circ}$, and $315^{\circ}$ were very similar to those at these symmetric positions $135^{\circ}, 90^{\circ}$, and $45^{\circ}$, waveforms only at these five positions were shown in this figure. These SAFE calculations agree well with experiments not only in amplitude distribution and arrival time of waveforms, but also in phase, which proves that these calculations were carried out with sufficient accuracy.

Figure 5 shows circumferential profiles at a target position in the focusing experiments. Amplitudes and time delays were calculated for a target point of $L=400 \mathrm{~mm}, 0^{\circ}$ and of $\mathrm{L}=800 \mathrm{~mm}, 0^{\circ}$ (Table I). Large signals were detected at the target point of $0^{\circ}$ in the both cases. This shows that focusing phenomena occur at the target points given by the SAFE calculations and the focusing algorithm. Calculated circumferential profiles are also shown in Fig. 4, where the focusing phenomena were simulated using amplitudes and time delays, these results being visualized later. Main differences between experimental and calculated profiles were caused by the fact that little excitation signals from eight transducers were not all well-formed tone-burst identical signals. This hardware issue can be improved with little difficulty.

Furthermore, interesting knowledge is obtained by comparing the amplitudes and time delays given in Table I with the waveforms shown in Fig. 4. Amplitudes and time delays obtained from the waveforms in Fig. 5 are shown in Table II, where the waveform at $180^{\circ}$ is assumed to be standard. By adding $n T$ ( $n$ is integer and $T$ is a period; $3.45 \mu$ s for 290 $\mathrm{kHz}$ ) into these time delays, values in the lowest row in Table II were obtained. Amplitudes and time delays in Table I $L=800$ and Table II show very good agreement but signs of the time delays are opposite. This means that the focusing can be realized as following. First, waveforms are detected at the $N$ receivers, where a single source transducer is installed at a target point. Then, $N$ received signals are time reversed. In the next step, $N$ receivers are used as $N$ transmitters and time-reversed signals are excited at these transmitters. This results in the focusing at the target point where the single

TABLE II. Amplitude and time delay $t_{\text {delay }}$ obtained by waveforms in Fig. 3 and time-delay shift $t_{\text {delay }} \pm n T$.

\begin{tabular}{lrrrrc}
\hline \hline & Top, $0^{\circ}$ & \multicolumn{1}{c}{$45^{\circ}$} & \multicolumn{1}{c}{$90^{\circ}$} & $135^{\circ}$ & Bottom, $180^{\circ}$ \\
\hline Amplitude [-] & 0.64 & 0.66 & 0.68 & 0.30 & 1.00 \\
Time delay [ $\mu \mathrm{s}] t_{\text {delay }}$ & -5.21 & 4.41 & -1.30 & 1.98 & 0.00 \\
$t_{\text {delay }} \pm n T[\mu \mathrm{s}]$ & -1.76 & -2.49 & -1.30 & -1.52 & 0.00 \\
\hline \hline
\end{tabular}


transducer was installed first. This is nothing other than the application of ultrasonic time reversal. ${ }^{22,23}$

\section{VISUALIZATION OF FOCUSING TECHNIQUE}

Since this focusing technique is based on the algorithm using complicated circumferential profiles, actual wave propagation status is hard to describe. For example, if focal points exist at unwanted points, they prevent us from determining the location of a crack. In order to apply the focusing technique to crack detection and defect evaluation, we need to comprehend wave motions and focusing status. In this section, the focusing technique was investigated by way of visualization results from SAFE calculations.

Figures 6(a) and (b) show the focusing status when amplitudes and time delays were tuned for focusing at $L$ $=400 \mathrm{~mm}, 0^{\circ}$ and $L=800 \mathrm{~mm}^{\circ}$, respectively. Visualization regions are from $20 \mathrm{~mm}$, just after excitation region, to 1000 $\mathrm{mm}$, where the grid width in the longitudinal direction is 10 $\mathrm{mm}$. The shift of grid points describes amplitudes of the wave and the shade is normal stress in the longitudinal direction. Circle charts in these figures represent circumferential profiles at the maximum amplitude position. As amplitudes and time delays were calculated for the harmonic wave of $290 \mathrm{kHz}$, modes were selected by controlling incident angle, in this case $\mathrm{L}(0,2)$ and its neighbor $\mathrm{F}(n, 2)$ modes, designed to focus to a focal point. However, it is possible to excite the other unwanted modes due to a frequency bandwidth of the incident wave with finite width transducers. Two wave packets can be seen in both Figs. 6(a) and (b), the faster one being the $\mathrm{L}(0,2)$ and $\mathrm{F}(n, 2)$ modes and the second being the $\mathrm{L}(0,1)$ and $\mathrm{F}(n, 1)$ modes. In both $\mathrm{L}=400 \mathrm{~mm}$ and $\mathrm{L}=800 \mathrm{~mm}, \mathrm{~L}(0,2)+\mathrm{F}(n, 2)$ modes are focused to the focal point as expected, while $\mathrm{L}(0,1)+\mathrm{F}(n, 1)$ modes are not.

Figures 6(a) and (b) reveal that the focusing phenomenon does not occur abruptly at the focal point, but goes through the process in which the first wave gradually increases as it approaches the focal point and decreases after the focal point. Considering the focusing algorithm, complex displacement amplitudes at the target position are described as $\mathbf{q}=\left[\begin{array}{lll}1 & 0 & 0, \ldots, 0\end{array}\right]^{\mathrm{T}}$. This means that the focused waves propagate after focusing like those excited by a single transducer at the focal point. In the vicinity of the focal points, circumferential profiles shows that waves spread with distance, while in the far field, complicated circumferential profiles which cannot be predicted without the aid of calculations are shown in Fig. 6(a) $t=220 \mu \mathrm{s}$. As for the wave before the focusing points, the time-reversal idea is helpful in understanding that the wave propagation is roughly symmetric with respect to the focal point. As seen in Fig. 6(a) $t=100 \mu \mathrm{s}$ and (b) $t=140 \mu \mathrm{s}$, circumferential profiles are similar to those for a single transmitter in the near field, that is, waves spread with distance from the target, but profiles became more complicated in far field as in Fig. 6(b) $t$ $=180 \mu \mathrm{s}$. From a crack detection point of view, even if unexpected focusing phenomena occur in the far field from the designed focal point, they do not result in a wrong evaluation of defects since these focal points are not close to each other, anyway.

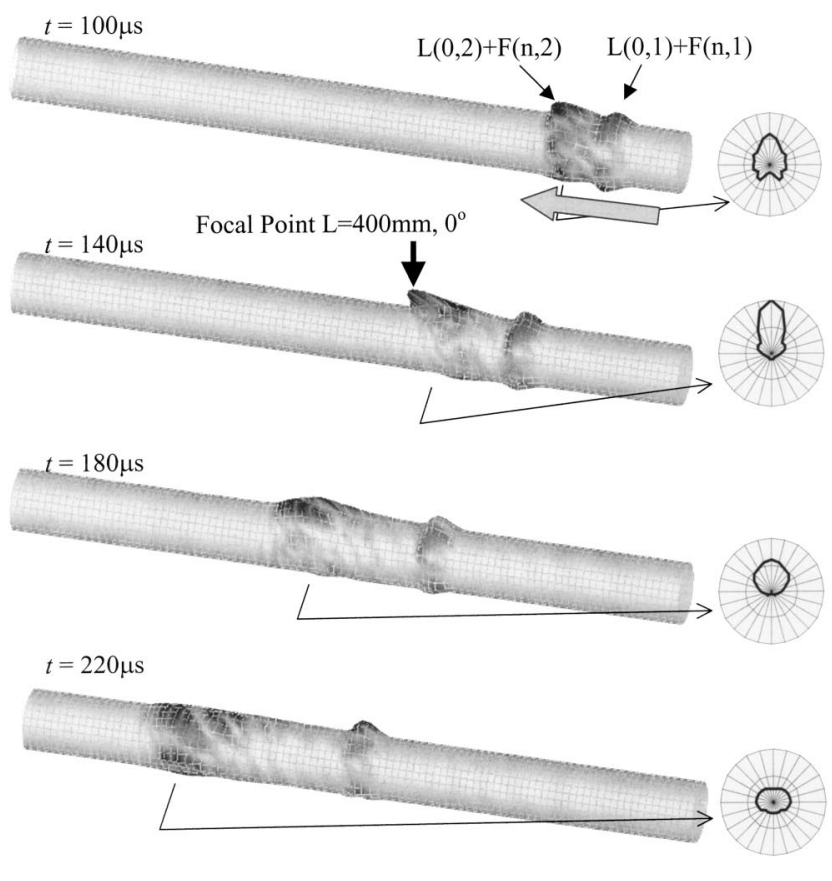

(a) Focal point $\mathrm{L}=400 \mathrm{~mm}$
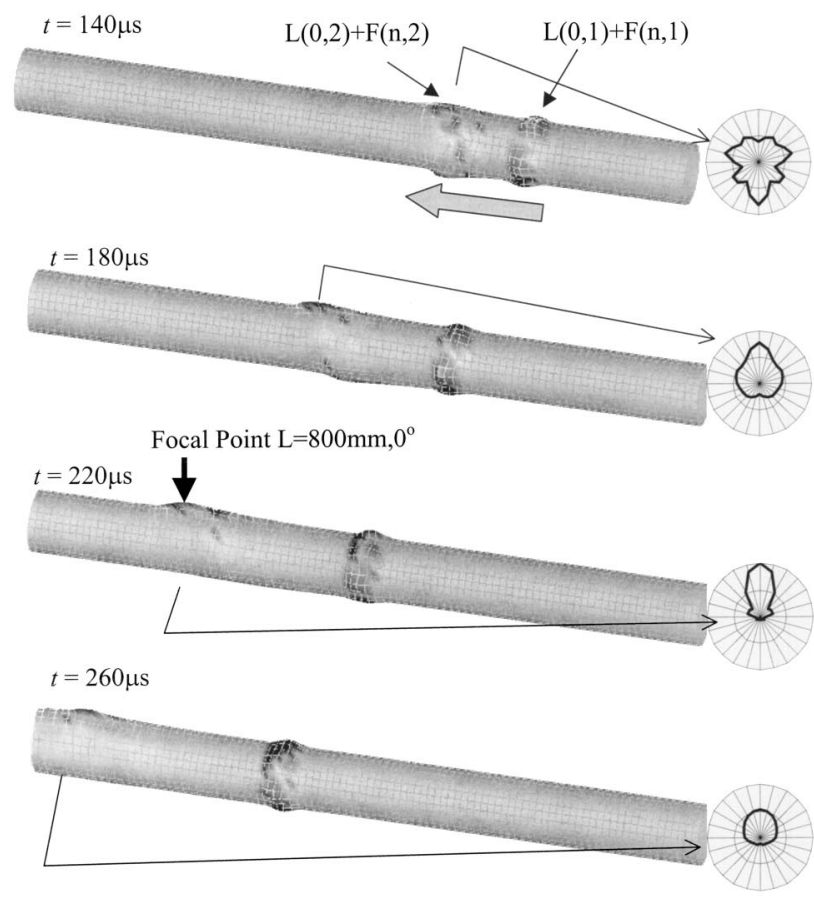

(b) Focal point $\mathrm{L}=800 \mathrm{~mm}$

FIG. 6. Visualization results for focusing status. Wave propagation on the pipe surface and circumferential profiles at the location of $\mathrm{L}(0,2)+\mathrm{F}(n, 2)$ modes.

\section{POTENTIAL OF THE FOCUSING TECHNIQUE}

Focal spot size strongly affects the sensitivity of lateral resolution in crack detection. To make a focal spot small, the following ideas are considered: (1) to increase the number of channels; (2) to control incident waves by function generators; (3) to change the location of the transducers.

The focusing algorithm gives some explanations of the 


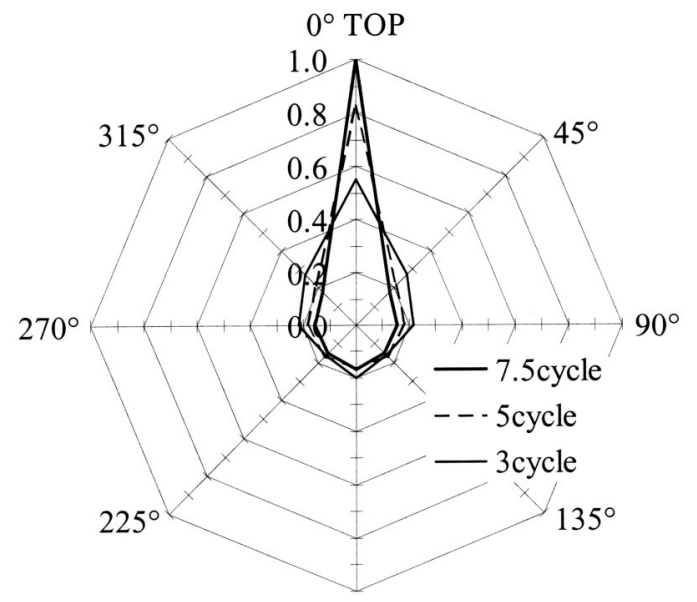

$180^{\circ}$ BOTTOM

FIG. 7. Circumferential profiles for incident waves with different frequency bandwidth and cycles of tone-burst waves.

resolution with regard to the number of channels. As the number of channels $N$ is large, the number of elements in vector $\mathbf{q}$ becomes large. This means that the circumferential width covered by one receiver becomes small, and this results in a small focusing spot. Detailed descriptions are written in Ref. 4.

Next, let us consider the control of incident waves. We discussed the use of tone-burst waves for focusing, in which amplitudes and time delays were obtained from a predicted circumferential profile by SAFE calculations only for a certain frequency $\omega$ (usually center frequency). However, if the circumferential profiles abruptly vary with a small shift of frequency $d \omega$, amplitudes and time delays obtained for a certain frequency $\omega$ are not designed to focus a wave of the frequency $\omega+d \omega$. Therefore, focus width could not be obtained as expected even in noise-less computer simulations. Figure 7 shows circumferential profiles at the focusing position with variable frequency bandwidth (number of cycles). As the number of cycles becomes small, that is, frequency bandwidth becomes broad, energy convergence at the focal point become small. This shows that amplitudes and time delays calculated for a center frequency do not work well for a converging ultrasonic wave at frequencies other than the center frequency.

For this reason, focusing is enhanced by applying amplitudes and time delays obtained independently for all frequency steps. Applying different amplitudes and time delays for different frequency steps means that arbitrary incident waves are excited instead of the tone-burst wave. From the time-reversal point of view, ${ }^{21,22}$ this can be replaced as follows. $N$ signals emitted from a single transducer and detected at $N$ receivers are converted into $N$ time-reversal waves. When the time-reversal waves are emitted back into the pipe from the $N$ transducers, the ideal focusings that are not influenced by frequency bandwidth like that in Fig. 7 are realized. Figure 8 shows received waves (calculation) at $\mathrm{L}=800$ $\mathrm{mm}$ when time reversal of calculated waveforms of the gray lines in Fig. 4 is used as excitation signals at eight transducers. Large waveforms were observed only at the desired focal

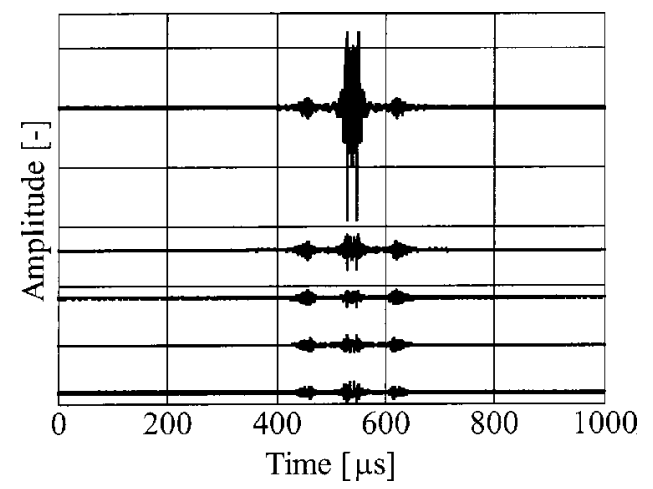

FIG. 8. Received signals for time-reversal emission tests (calculation) at the focal point $\left(\mathrm{L}=800 \mathrm{~mm}, 0^{\circ}\right)$.

point $\left(\mathrm{L}=800 \mathrm{~mm}, 0^{\circ}\right)$, and small amplitudes at the other points.

Next, let us discuss the transducer location. The focusing algorithm can be applied even if transducers are not aligned in the circumferential direction. It is possible that changing the location of the transducers could improve focusing. However, since it is not easy to find the general algorithm on transducer location to optimize focusing, qualitative discussions will be described here. When the number of channels are $N$, the above-mentioned discussions are applied in this case, and the resolution does not change. But, the robustness of the solution $\mathbf{H}^{-1}$ is affected, and it results in a poor focusing characteristic. For example, when $N$ transducers are at the same position, this is the same situation as a single transducer, and so focusing is not observed. Here, the matrix $\mathbf{H}$ is nonregular as $H_{11}=H_{21}=\cdots H_{N 1}, H_{12}=H_{22}$ $=\cdots H_{N 2}, \quad H_{13}=H_{23}=\cdots H_{N 3}, \ldots, \quad H_{1 N}=H_{2 N}=\ldots H_{\mathrm{NN}}$. Generally speaking, the robustness of Eq. (14) depends on the independence of each equation in the simultaneous equations Eq. (14). In this sense, aligning transducers in the circumferential direction at a regular interval is the best arrangement to keep independence of these simultaneous equations and to give good focusing.

\section{CONCLUSIONS}

Simulation of guided wave propagation was conducted using a semianalytical finite element method. This calculation was verified by way of a comparison of waveforms and an application to the focusing technique. Applying the semianalytical FEM calculations to focusing technique gave the following conclusions.

(1) Focusing does not occur abruptly at the focal point, but the amplitude of a wave packet increases as it approaches the focal point and decreases gradually after it. In the far field from the focal point, circumferential profiles become complicated. This is just like circumferential profiles from a single source at the focal point.

(2) Controlling incident waveforms, as well as increasing the number of channels, improves the resolution and sensitivity of the focusing technique. Using time-reversal signals realizes the ideal focusing in a pipe.

In this paper, circumferential resolutions in the focusing 
technique were discussed, but it is necessary to investigate the resolution in the longitudinal direction, for which visualization will be helpful.

\section{ACKNOWLEDGMENTS}

We would like to thank Plant Integrity Ltd., Cambridge, England and Peter Mudge for their encouragement and partial support of this work on focusing in utilizing the nonaxisymmetric pipe modes.

${ }^{1}$ J. J. Ditri and J. L. Rose, "Excitation of guided elastic wave modes in hollow cylinders by applied surface tractions," J. Appl. Phys. 72(7), 2589-2597 (1992).

${ }^{2}$ H. J. Shin and J. L. Rose, "Guided waves by axisymmetric and nonaxisymmetric surface loading on hollow cylinders," Ultrasonics 37, 355-363 (1999).

${ }^{3} \mathrm{~J}$. Li and J. L. Rose, "Excitation and propagation of nonaxisymmetric guided waves in a hollow cylinder," J. Acoust. Soc. Am. 109(2), 457-468 (2001).

${ }^{4} \mathrm{~J}$. Li, "Quantitative guided wave evaluation," Ph.D. thesis, The Pennsylvania State University (2001).

${ }^{5}$ J. L. Rose and X. Zhao, "Flexural mode tuning for pipe elbow testing," Mater. Eval. 59(5), 621-624 (2001).

${ }^{6}$ Z. Sun, J. L. Rose, M. Quarry, and D. Chinn, "Flexural mode tuning in pipe inspection," in Review of Progress in Quantitative NDE, edited by D. O. Thompson and D. E. Chimenti (Plenum, New York, 2002), Vol. 21, pp. 262-269.

${ }^{7}$ J. L. Rose, "Guided Wave Ultrasonic Pipe Inspection-The Next Generation," Proceedings of the 8th ECNDT, Barcelona, Spain (2002).

${ }^{8}$ D. N. Alleyne, M. J. S. Lowe, and P. Cawley, "The reflection of guided waves from circumferential notches in pipes," J. Appl. Mech. 65, 635641 (1998).

${ }^{9}$ R. Long, K. Vine, M. J. S. Lowe, and P. Cawley, "Monitoring acoustic wave propagation in buried cast iron water pipes," in Review of Progress in Quantitative NDE, edited by D. O. Thompson and D. E. Chimenti (Plenum, New York, 2001), Vol. 20, 1202-1209.
${ }^{10}$ P. Wilcox, M. Lowe, and P. Cawley, "The effect of dispersion on longrange inspection using ultrasonic guided waves," NDT \& E Int. 34, 1-9 (2001).

${ }^{11}$ D. N. Alleyne, B. Pavlakovic, M. J. S. Lowe, and P. Cawley, "Rapid, long-range inspection of chemical plant pipework using guided waves," in Review of Progress in Quantitative NDE, edited by D. O. Thompson and D. E. Chimenti (Plenum, New York, 2001), Vol. 20, pp. 180-187.

${ }^{12}$ D. C. Gazis, "Three-dimensional investigation of the propagation of waves in hollow circular cylinders. I. Analytical foundation. II. Numerical Results," J. Acoust. Soc. Am. 31, 568-578 (1959).

${ }^{13}$ Y. K. Cheung, Finite Strip Method in Structural Mechanics (Pergamon, Oxford, 1976).

${ }^{14}$ O. C. Zienkiewicz and R. L. Taylor, The Finite Element Method, 5th ed. (Butterworth-Heinemann, Washington, D.C., 2000).

${ }^{15}$ S. K. Datta, A. H. Shah, R. L. Bratton, and T. Chakraborty, "Wave propagation in laminated composite plates," J. Acoust. Soc. Am. 83, 2020-2026 (1988).

${ }^{16}$ W. Karunasena, A. H. Shah, and S. K. Datta, "Wave propagation in a multilayered laminated cross-ply composite plate," ASME Trans. J. Appl. Mech. 58, 1028-1032 (1991).

${ }^{17}$ T. Kohl, S. K. Datta, and A. H. Shah, "Axially symmetric pulse propagation in semi-infinite hollow cylinders," AIAA J. 30(6), 1617-1624 (1992).

${ }^{18}$ N. Rattanawangcharoen, A. H. Shah, and S. K. Datta, "Reflection of waves at the free edge of a laminated circular cylinder," J. Appl. Mech. 61, 323-329 (1994).

${ }^{19}$ W. Zhuang, A. H. Shah, and S. B. Dong, "Elastodynamic Green's function for laminated anisotropic circular cylinders," J. Appl. Mech. 66, 665-674 (1999).

${ }^{20}$ H. Taweel, S. B. Dong, and M. Kazic, "Wave reflection from the free end of a cylinder with an arbitrary cross section," Int. J. Solids Struct. 37, 1701-1726 (2000).

${ }^{21}$ T. Hayashi and K. Kawashima, "Multiple reflections of Lamb waves at a delamination," Ultrasonics 40, 193-197 (2002).

${ }^{22}$ M. Fink, "Time reversal of ultrasonic fields. I. Basic principles," IEEE Trans. Ultrason. Ferroelectr. Freq. Control 39(5), 555-566 (1992).

${ }^{23}$ R. K. Ing and M. Fink, "Time-reversed Lamb waves," IEEE Trans. Ultrason. Ferroelectr. Freq. Control 45(4), 1032-1043 (1998). 